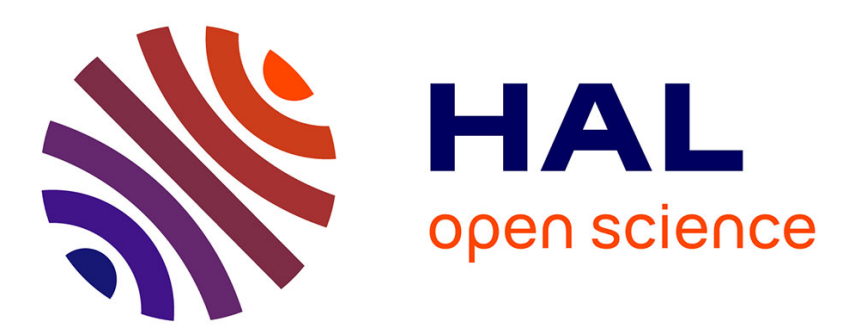

\title{
Insect-inspired omnidirectional vision for autonomous localization on-board a hexapod robot
}

Julien Dupeyroux, Sean Lapalus, Ilya Brodoline, Stéphane Viollet, Julien

Serres

\section{- To cite this version: \\ Julien Dupeyroux, Sean Lapalus, Ilya Brodoline, Stéphane Viollet, Julien Serres. Insect-inspired omnidirectional vision for autonomous localization on-board a hexapod robot. 28th Mediterranean Conference on Control and Automation (MED), 2020 IEEE International Conference on, Sep 2020, Saint-Raphaël, France. 10.1109/MED48518.2020.9183091 . hal-02873396}

\section{HAL Id: hal-02873396 \\ https://hal-amu.archives-ouvertes.fr/hal-02873396}

Submitted on 18 Jun 2020

HAL is a multi-disciplinary open access archive for the deposit and dissemination of scientific research documents, whether they are published or not. The documents may come from teaching and research institutions in France or abroad, or from public or private research centers.
L'archive ouverte pluridisciplinaire HAL, est destinée au dépôt et à la diffusion de documents scientifiques de niveau recherche, publiés ou non, émanant des établissements d'enseignement et de recherche français ou étrangers, des laboratoires publics ou privés. 


\title{
Insect-inspired omnidirectional vision for autonomous localization on-board a hexapod robot*
}

\author{
Julien Dupeyroux, Sean Lapalus, Ilya Brodoline, Stéphane Viollet and Julien R. Serres
}

\begin{abstract}
Navigating insects like desert ants are known to robustly estimate their position from their nest while foraging for food, for several hundred meters across hostile environments, by means of very low resolution visual information processing. This tour de force stands for a great source of inspiration to design smart, parsimonious and robust solutions to make robots of any size navigate in global navigation satellite systems-denied or in cluttered environments. In this study, we introduce a new insect-inspired omnidirectional visual sensor $(640 \times 120$ pixels; 120 fps $)$. The inter-pixel angle is equal to $0.6^{\circ}$ and the acceptance angle is equal to $1.5^{\circ}$, which is comparable to those observed in predatory flying insects. This sensor was embedded on-board the AntBot robot, a six-legged walking robot mimicking desert ants at morphological, locomotive and sensing levels. Despite the residual visual oscillations of the field of view while walking, the robot successfully detected fixed obstacles and was able to locate itself with an accuracy as low as $25 \pm 10 \mathrm{~cm}$, which actually corresponds to an average error of only 3 strides (hexapod stride length: $8.2 \mathrm{~cm}$ ) after a $9 \mathrm{~m}$-long journey. This suggests that low-acuity visual sensors, inherently requiring few computational resources, are good candidates for ant-like familiarity-based navigation in cluttered environments.
\end{abstract}

\section{INTRODUCTION}

Autonomous navigation is one of the leading contemporary technological challenges for which the potential applications are many and various, in both mobile robotics and means of transport. Among the current state-of-the-art, the widely distributed civilian Global Navigation Satellite System (GNSS) has yielded strong performances in urban environments. Yet, the accuracy $(5-30 \mathrm{~m})$ of the GNSS heavily depends on the meteorological conditions and the urban infrastructures. Computer vision based methods also provide localization cues for autonomous vehicles in highly complex environments. However, data processing requires important computational resources, and light changes often result in navigation failure. The challenge here is to develop an alternative navigation system that could be combined with conventional techniques so that any vehicle equipped with such solution would benefit from high precision and robust navigation skills in more complex environments.

In recent years, we have developed an ant-inspired walking robot, called AntBot (Fig. 1) [1], endowed with parsimonious visual sensors mimicking the compound eye of the desert

*This research was supported by the French Direction Générale de l'Armement (DGA), CNRS, Aix Marseille University, the Provence-AlpesCôte d'Azur region, and the French National Research Agency for Research (ANR) with the Equipex/Robotex project.

All authors are with the Biorobotics Team, Aix Marseille Univ, CNRS, ISM, Marseille, France. julien. serresduniv-amu. fr ants Cataglyphis fortis [2]. AntBot is equipped with a 2pixel celestial compass that detects the state of polarization of the skylight in the ultraviolet (UV) range [3]. This minimalist sensor provides an optical heading while navigating outdoor with excellent performances regardless of environmental conditions (cloud cover, haze, UV-index) [4], [5]. Besides, AntBot comprises an optic flow (OF) sensor, called the $\mathrm{M}^{2} \mathrm{APix}$ sensor (Michaelis-Menten Auto-adaptive Pixels) [6]. This sensor includes 12 pixels that auto-adapt up to 7 decades light changes just like photoreceptors in vertebrates and invertebrates [7]. We combined ventral OF measurements with stride counting to allow AntBot estimating its walked distance.

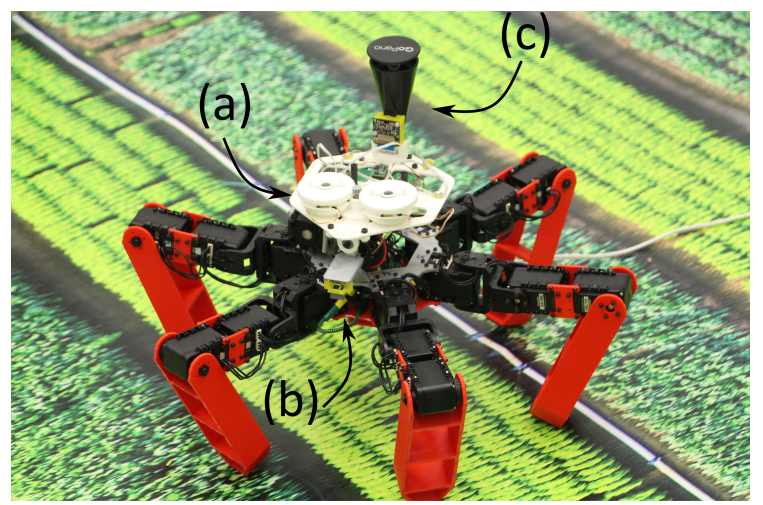

Fig. 1. The AntBot robot (mass: $2.3 \mathrm{~kg}$, diameter: $45 \mathrm{~cm}$ ). (a) The celestial compass for the detection of the optical heading based on the polarization pattern of UV skylight. (b) The $\mathrm{M}^{2} \mathrm{APix}$ sensor for the OF-based distance estimation. (c) The low-resolution omnidirectional vision sensor for obstacle avoidance and snapshot-based localization, used alone in this study.

According to the desert ants navigational toolkit [8], the path integrator (PI) merges polarization cues (orientation) and $\mathrm{OF}$ cues (distance) to estimate the localization with respect to the departure point. This strategy has been successfully tested on-board AntBot, resulting in a homing error of $6.7 \mathrm{~cm}$ on average, which is lower than one robot's stride $(8.2 \mathrm{~cm})$, after a $15 \mathrm{~m}$-long journey [1]. In its current version, AntBot is able to navigate autonomously in unknown outdoor environments regardless of the meteorological conditions. However, the PI approach is prone to cumulative error as trajectory length increases. In addition to PI, desert ants rely on visual-based navigation, i.e. visual guidance (VG) [9], [10], [11]. For example, VG allows ants to return directly to familiar terrain from novel locations [12]. VG helps foraging insects to find their way home by comparing memorized snapshots to the current view of the scene. This strategy is 
mostly used when the insect is roaming in cluttered environments, as for the Australian desert ants Melophorus bagoti [11]. In such a context, ants are expected to encounter several trees, tussocks and rocks prominent enough to be further used as visual landmarks for navigation purposes (odometry, orientation) [13]. Although the relationship between VG and PI remains unknown, it has been shown that both of them can be active simultaneously [9], [11], and that PI is never switched off [8]. Desert ants memorize a series of snapshots along routes towards the nest [10]. These routes mark out the so-called catchment area [14], [15]. The insect compares what it sees with the images it has memorized. This comparison provides a course to follow by angular difference of the omnidirectional images. As the insect approaches the nest, there is a decrease in image difference [16].

In this study, two additional ant-inspired navigation skills have been implemented on-board the AntBot robot based on low-acuity omnidirectional visual sensor: (i) OF-based obstacle avoidance, and (ii) snapshot-based autonomous localization. This study is structured as follows: section II provides a brief overview of related work on visual-based navigation applied to autonomous vehicles. The insect-inspired omnidirectional vision sensor is introduced in section III. Then, obstacle avoidance is described in section IV, and snapshotbased navigation in section $\mathrm{V}$.

\section{RELATED WORK}

Over the past decades, omnidirectional vision [17] in the context of localization has received significant attention. In the late 1990s, Lambrinos et al. worked on the development of a desert ant-inspired wheeled robot, called Sahabot 2 [18]. This robot was equipped with a sky compass for the estimation of its heading. It also included a wheel encoder for the odometric estimation, and an omnidirectional vision system. The snapshots were reduced to 1-D and used for determining the homing vector direction of the robot. This was performed by comparing the current retinal position of landmarks to the 1-D omnidirectional image taken at the goal location. Later, Stürzl and Mallot introduced a method based on the Fourier transform to estimate the orientation of their ground robot Khepera equipped with an omnidirectional vision sensor [19]. Images were reduced to 1-D with a resolution of $1^{\circ}$ per pixel. Their approach successfully addressed key elements like the memory usage and computational requirements for embedded applications. Very promising results have been suggested on-board the HECTOR hexapod robot lately with a frontal fisheye lens [20]. Further studies made use of omnidirectional vision to help determining topological maps [21], [22] and converging towards SLAM strategies [23], [24]. Recent studies have shown new opportunities coming with the event-based cameras, as reported in [25], since they offer multiple advantages over regular cameras, like asynchronous processing of motion, high temporal resolution (few $\mu$ s latency), and limited sensitivity to light variations.

Other methods for image processing and localization are based on neural approaches. In 2000, Gaussier et al. pro- posed a neural network based on the PerAc (PerceptionAction) architecture to solve visual-based homing with a wheeled robot [26]. According to this model, the robot was able to learn different places and connect them to each others to create a sort of cognitive map. More recently, Ran et al. trained a convolutional neural network to predict the possible path directions of a ground robot equipped with a non-calibrated omnidirectional vision sensor [27]. Neuralbased strategies are inherently robust to modification of the scene, like the addition, the displacement or the removal of objects (i.e. visual landmarks). In that respect, the fast developing field of spiking neural networks, particularly when coupled with event-based cameras, will definitely make super-efficient and optimized neuromorphic solutions for omnidirectional vision-based autonomous navigation onboard small ground robots, or even flying drones.

Omnidirectional vision in the context of autonomous localization has mostly been tested on-board wheeled robots. The resolution of the sensors was, for the vast majority, significantly much higher than in the insects' compound eye. Consequently, it would be of great interest to explore the possibilities of autonomous localization on-board an insectoid robot that is intrinsically prone to oscillatory visual disturbances, and with a coarse visual resolution - which will be further referred as visual acuity - similar to insects' compound eye. Such an omnidirectional visual sensor will make it possible to test insect-based VG models, especially desert ants, by combining PI navigation and snapshot-based localization on-board the AntBot robot.

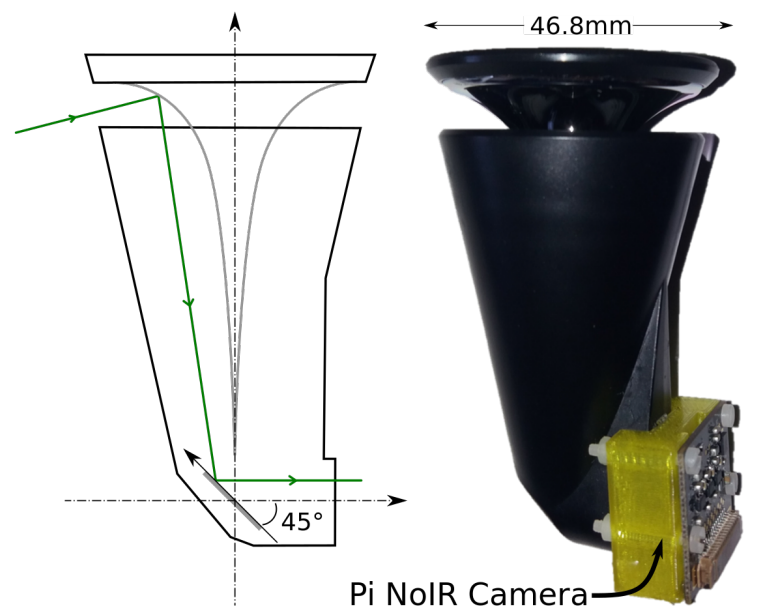

Fig. 2. The GoPano ${ }^{\mathrm{TM}}$ omnidirectional lens mounted on the Raspberry Pi NoIR camera. The mirrors are displayed in grey. An example of light pathway through the omnidirectional lens is shown in green.

\section{INSECT-INSPIRED LOW-ACUITY OMNIDIRECTIONAL VISION}

The omnidirectional visual sensor is embedded on top of the AntBot robot (Fig. 1), with an overall weight of 32.4 $\mathrm{g}$; it is composed of a Pi NoIR camera ( 8 megapixels; up to $120 \mathrm{fps}$, Fig. 2) on which we mounted the GoPano ${ }^{\mathrm{TM}}$ lens, consisting of a curved mirror that reflects a panoramic 

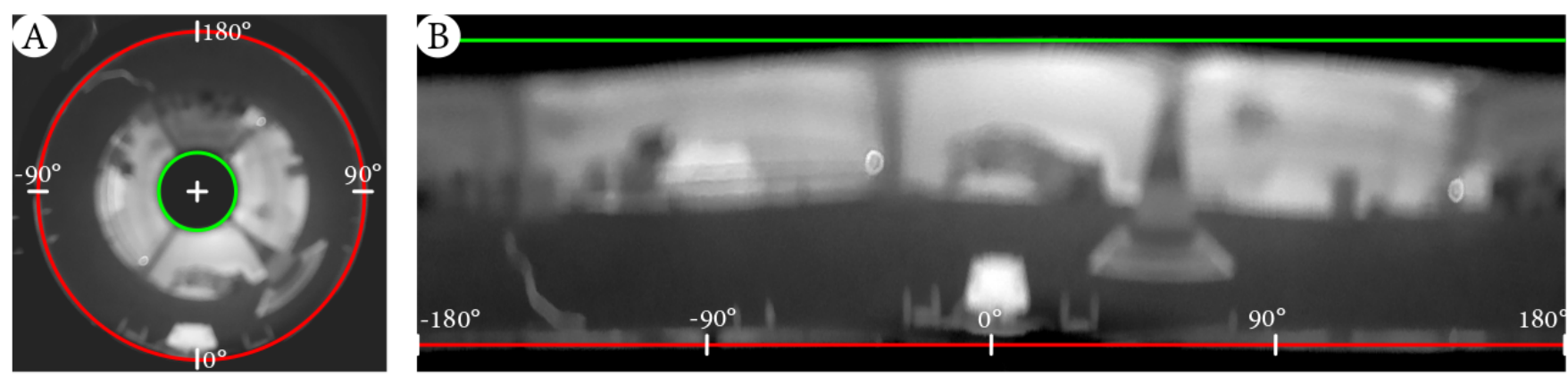

Fig. 3. Example of a snapshot captured with the omnidirectional visual system. A Original picture where the margin circles were detected using the Hough transform. B Unwrapped snapshot showing the $360^{\circ}$ omnidirectional view in our flying arena of the Mediterranean.

view to the camera. An example of panoramic snapshot is presented in Fig. 3A.

We reproduce here the visual acuity, i.e. the interommatidial angle $\Delta \phi$, of insects like honeybees (1.9 ${ }^{\circ}$ [28]), flies $\left(1.5^{\circ}[29]\right)$ and desert ants $\left(4^{\circ}[30]\right)$, which is known to fluctuate depending on both the size and the type of compound eye, but also on many other aspects including, for example, whether the insect is a predator or not (see review [31]). This was performed by increasing the distance between the optical center of the camera, and the omnidirectional lens. Therefore, the focal points of each lens (i.e. camera lens and panoramic lens) are separated and introduce analog blur, i.e. reduce the visual acuity.

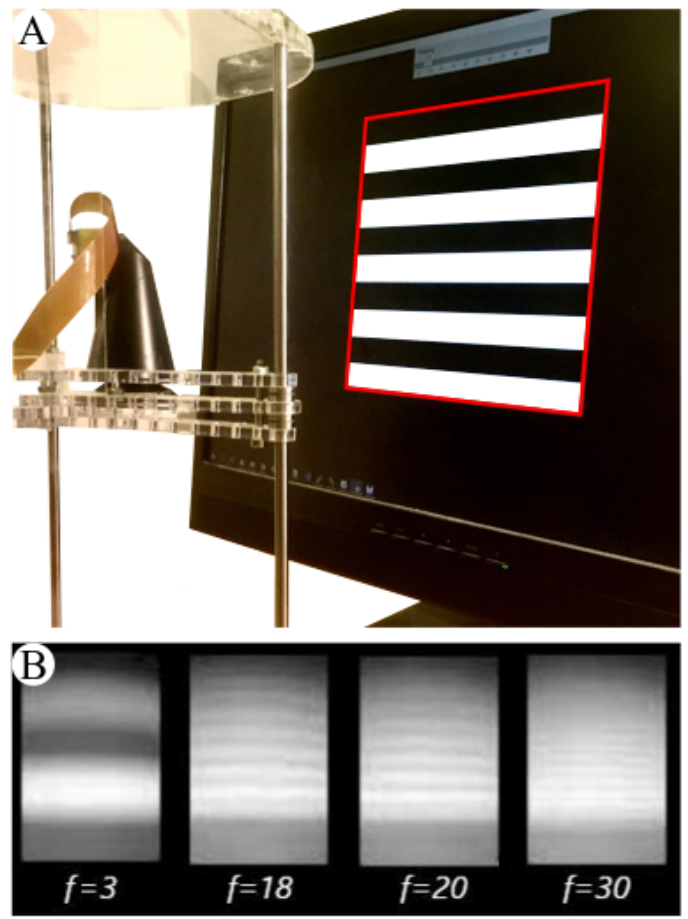

Fig. 4. Characterization of the omnidirectional sensor's visual acuity. A Experimental setup showing the sensor in front of the monitor displaying the calibration pattern. B Examples of pictures for varying spatial frequencies. $f$ represents the number of cycles.

The visual acuity $V . A$. of an optical system is defined as the minimal resolution angle, and is inversely proportional to the spatial frequency $\nu$ (in cycles $/{ }^{\circ}$ ). We can find the spatial cut-off frequency $\nu_{c o}$ of the optical system in its Modulation Transfer Function (MTF) [31]:

$$
V . A .=\Delta \rho=\frac{1}{\nu_{c o}}
$$

The experimental setup for the characterization of the visual acuity (Fig. 4A) was composed of the omnidirectional visual sensor placed in front of a square target $(20 \times 20$ $\mathrm{cm}$ ) at a distance of $23 \mathrm{~cm}$. The target consisted of a series of white-and-black stripes with varying spatial frequency and displayed by a standard monitor. Multiple pictures were taken with the omnidirectional sensor, and further processed to determinate its acuity. During acquisitions, the position of the camera remained fixed; only the spatial frequency $f$ was changed. The procedure consisted in taking pictures while increasing $f$ until it became impossible to distinguish two consecutive lines (i.e., MTF $=0$ ). Resulting examples are plotted in Fig. 4B. The target's frequency threshold was found to be equal to $f_{t h}=31$ cycles. Therefore, the sensor's cut-off spatial frequency $\nu_{c o}$ can be computed as follows:

$$
\nu_{c o}=\frac{f_{t h}}{2 \cdot \gamma} \approx 0.66 \text { cycles }{ }^{\circ}
$$

where $\gamma=\operatorname{atan}((20 / 2) / 23)=23.5^{\circ}$ is the angular diameter of the target in degrees. The visual acuity V.A. is therefore equivalent to an acceptance angle $\Delta \rho$ of $1.5^{\circ}$ (Eq. 1), which is consistent with the ones of insects' compound eyes, and thus confirms the suitability of our omnidirectional sensor as a robotic artifact of the visual system of insects.

An example of the snapshots recorded by the omnidirectional camera is provided in Fig. 3A. By applying a double Hough transform, the two margin circles were detected and used in a custom-made unwrapping program (Fig. 3B). For all the applications, pictures were captured with a resolution of $640 \times 480$ pixels, and the resolution of the resulting unwrapped snapshot was of $640 \times 120$ pixels (i.e. 76,800 pixels), then providing an inter-pixel angle of $\Delta \phi=0.6^{\circ}$.

\section{OF-BASED DETECTION OF OBSTACLES}

The computation of the OF was performed on the unwrapped snapshots by means of the Lucas-Kanade method 
implemented within the OpenCVTM graphics programming environment [32], [33]. As far as we know, the pyramidal implementation of the Lucas-Kanade method is the fastest algorithm in image processing to extract the OF. According to the Lucas-Kanade method, we assume that the displacement of objects in two consecutive snapshots is small and approximately constant within a neighborhood of a selected pixel. This hypothesis is consistent with the robot's speed that was set at $10 \mathrm{~cm} / \mathrm{s}$, producing small OF with respect to both the visual acuity of the omnidirectional visual sensor and the configuration of the environment (Fig. 5).

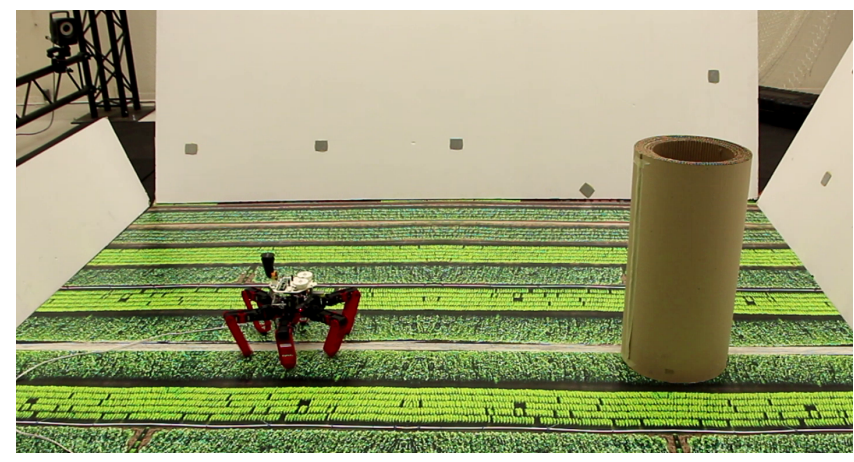

Fig. 5. The AntBot robot is walking straight forward in the direction of the obstacle (i.e. cylinder).

A set of $N$ points of interests (PoI) were first identified for each snapshot within a predefined region of interest (RoI), i.e. a fixed fraction of the field of view (FoV) centered around the horizon line (e.g., middle of the FoV). Then, the matching PoI between two consecutive snapshots found, thus providing an estimate of the displacement vector used for the computation of the OF. To limit the noise, only the PoI for which the estimated OF was higher than an arbitrarily fixed threshold value were kept.

Two examples of the results we obtained are displayed in Fig. 6. For each snapshot, the histogram of the selected PoI producing $\mathrm{OF}$ vectors were plotted to help the visualization of clusters. These clusters were considered as potential obstacles to avoid. On Fig. 6A, results show the PoI and clusters within a RoI set at $100 \%$ of the Fov, with $N=1000$. These parameters led to the detection of the frontward obstacle, but also the motion of the ground and the legs, therefore considered as obstacles to avoid. To prevent from such a mistake, the number of PoI was decreased to $N=100$, and the RoI was set at $30 \%$ of the FoV, thus removing the OF caused by the robot's legs (see Fig. 6B). Besides, the OF threshold was slightly increased. The results are shown in Fig. 6B, where only the frontward obstacle is identified. In a navigational context, the angular sector depicted by the cluster of PoI can be used to set the new heading of the robot.

\section{SNAPSHOT-BASED LOCALIZATION}

In this section, the snapshot-based indoor localization was addressed under the low visual acuity constraint, including visual disturbances coming from the walk of the robot.

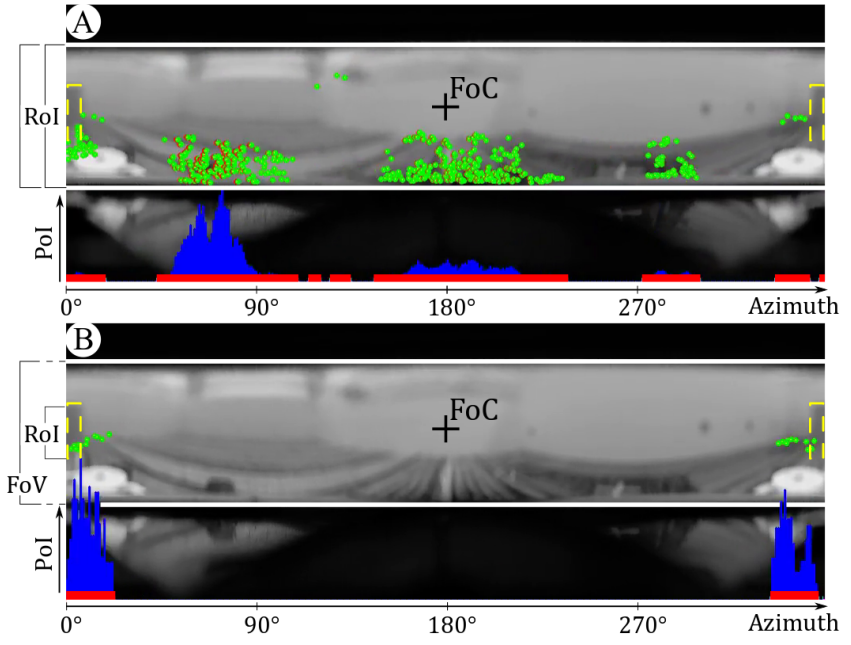

Fig. 6. Results obtained for the experimental context introduced in Fig. 5. A $N=1000$; RoI set at $100 \%$ of the FoV. B $N=100$; RoI set at $30 \%$ of the FoV. For each snapshot, the frontward obstacle is magnified by the dashed yellow box. FoC: focus of contraction, diametrically opposite to the focus of expansion (FoE). Thick red lines depict the clusters of PoI, i.e. the obstacles' angular, based on the PoI histogram (in blue).

Image normalization was not necessary in this experimental context since the light control prevented from any variation. The snapshot comparison method applied relied on the root means square (R.M.S.) difference of omnidirectional snapshots, as introduced in Zeil et al. [16], for which the visual acuity was of $0.2^{\circ}$. The following experiments were performed indoor, in the flying arena of the Mediterranean equipped with 17 motion-capture cameras (VICON ${ }^{\mathrm{TM}}$ ) covering a re-configurable space $(6 \times 8 \times 6 \mathrm{~m})$.

First, the robot was asked to walk over a $2 \mathrm{~m}$-long straight line and take a snapshot every $5 \mathrm{~cm}$. The R.M.S. image difference was computed between these views and a memorized snapshot taken at the center of the line (Eq. 3). This procedure provides us with an image difference value for each location along the line. This acquisition was repeated for two lighting conditions (artificial light), namely at 1200 and 120 Lux. Results are displayed in Fig. 7.

$$
\text { R.M.S. }=\sqrt{\frac{1}{m \times n} \sum\left(I(x, y)-I_{r e f}(x, y)\right)^{2}}
$$

These results are consistent with those presented in [16] as the difference function for a particular memorized snapshot was computed, showing typical shape and smooth variations with the distance to the reference location. The depth observed at the middle of the course proves that omnidirectional snapshot-based navigation relying on a simple R.M.S. image difference method, with coarse visual acuity $\left(1.5^{\circ}\right)$ and even low lighting conditions, is enough to reach a $5 \mathrm{~cm}$ localization accuracy, provided the initial grid mesh of memorized snapshots.

In a second series of tests, we had the robot navigating in the flying arena where boxes of different sizes and shapes were put on the floor to serve as visual landmarks for 


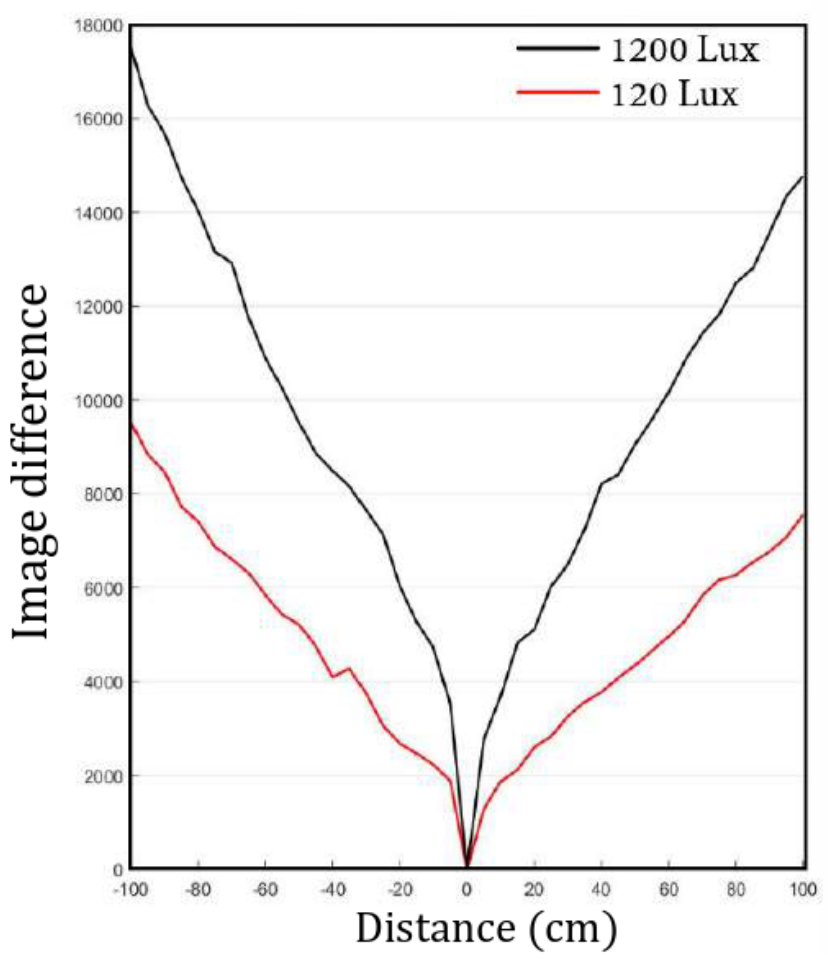

Fig. 7. Difference function (R.M.S.) from a reference location (at 0) along a straight line and for two distinct light conditions: 1200 Lux and 120 Lux.

localization (Fig. 8). Prior to the acquisition, a 2-D grid mesh of memorized snapshots were collected with $50 \mathrm{~cm}$ resolution along the y-axis, and 1m-resolution along the $\mathrm{x}$-axis. Each of the omnidirectional snapshots were taken with the same orientation. While navigating, the robot took snapshots of its current view at random times. The R.M.S. image difference was then used to determine to which node of the grid mesh the robot was the closest. To deal with the robot's changing heading, the current view was rotated by successive $90^{\circ}$ steps. Results show that for each actual position of the robot, the closest node (memorized snapshot) was successfully activated. On average, the distance error between the robot's actual location and the activated node snapshots reached $25 \pm 10 \mathrm{~cm}$ (mean $\pm s d$ ), with a median equal to $24 \mathrm{~cm}$ (Fig. 9Left). More precisely, the average error along the $\mathrm{x}$-axis was found to be equal to $16 \pm 11$ $\mathrm{cm}$ (median: $15 \mathrm{~cm}$ ), and $16 \pm 10 \mathrm{~cm}$ (median: $18 \mathrm{~cm}$ ) along the y-axis. These results are consistent with the 2$\mathrm{D}$ grid mesh resolution, though we would have expected a greater average error along the $\mathrm{x}$-axis. An example of image difference distribution for the 4th snapshot of the robot is displayed in Fig. 9Right.

\section{CONCLUSION}

In this study, we presented a series of visual-based navigation behaviors implemented on-board the AntBot hexapod robot using an omnidirectional visual sensor, the coarse visual acuity of which makes it similar to the insects' compound eye. The characterization of the omnidirectional

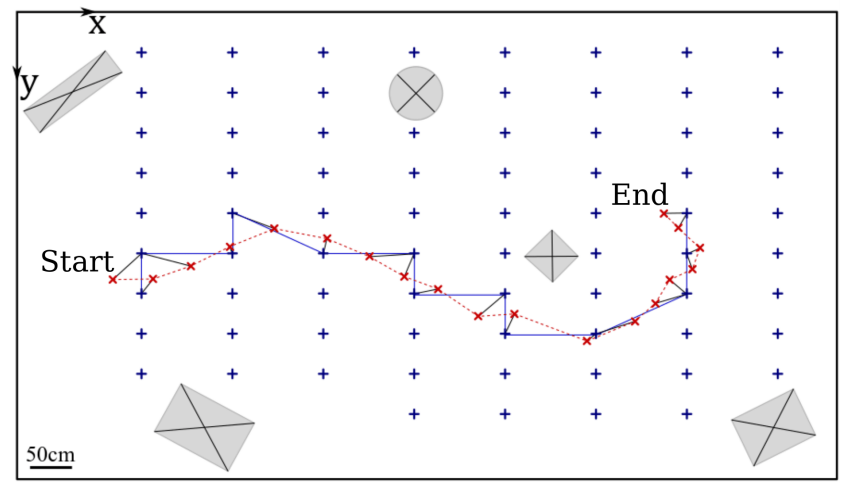

Fig. 8. Top view of the snapshot-based localization experiment in the flying arena of the Mediterranean. Blue markers depict the position of the memorized snapshots. Red markers show the actual location of the robot when the current snapshot was taken. The red dashed curve shows the real trajectory of the robot, and the blue curve shows the believed trajectory based on the memorized snapshots activated by the r.m.s. difference (black lines).
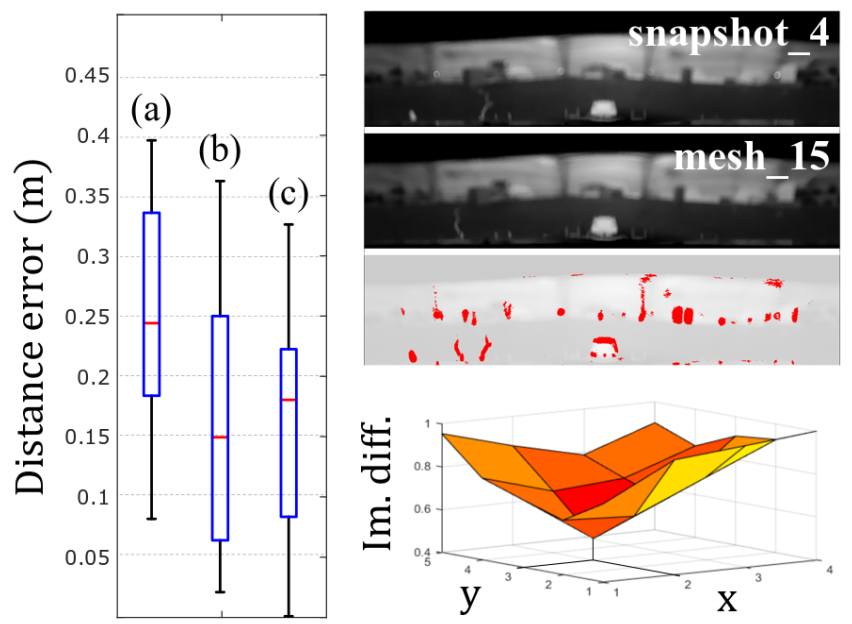

Fig. 9. Localization estimation results. Left Overall results showing the (a) 2-D distance, (b) x-axis, and (c) y-axis error distributions between the robot's believed position according to the 2-D grid mesh, and its actual location when taking pictures. Right Example of image difference between snapshot \#4 and node snapshot \#15 (the one activated by the R.M.S.; see Fig. 4). The bottom chart displays the image difference according to the neighboring node snapshots.

camera reported a coarse visual acuity equal to $1.5^{\circ}$, corresponding to 90 minutes of arc. As a comparison, the visual acuity of the human's eye is 1 minute of arc $\left(1^{\circ} / 60\right)$ in the fovea.

Obstacle detection was performed within a region of interest set at $30 \%$ of the total field of view, centered around the horizon line. AntBot was able to detect obstacles with a coarse acuity while walking, despite its locomotion mode which naturally causes oscillations of the field of view. Besides, autonomous localization was successfully performed within an indoor cluttered environment with visual landmarks (e.g. boxes), reaching an accuracy of $0.25 \mathrm{~m}$ after a $9 \mathrm{~m}$-journey and representing only 3 steps error on average (hexapod stride length: $8.2 \mathrm{~cm}$ ). 
These results demonstrate that high-resolution vision is not necessary to achieve complex tasks like homing. Further investigations will consider automatic detection of the FoE/FoC points, which should contribute to the optimization of the performance of detection of obstacles while walking. As for desert ants, AntBot could combine a path integration with a coarse ommidirectional vision to autonomously explore unknown environments while avoiding obstacles. The next question will be to understand how these two components of the insects' navigational toolkit merge together. Regarding this work and our prior studies on path integration [1], we believe that AntBot is a reliable robotic replica of desert ants which, besides leading to new parsimonious and accurate navigation systems for robotic applications, can be used as an experimental platform for testing biological models [1].

\section{REFERENCES}

[1] J. Dupeyroux, J. R. Serres, and S. Viollet, "Antbot: A six-legged walking robot able to home like desert ants in outdoor environments," Science Robotics, vol. 4, no. 27, 2019.

[2] R. Wehner and S. Wehner, "Insect navigation: use of maps or ariadne's thread?" Ethology Ecology \& Evolution, vol. 2, no. 1, pp. 27-48, 1990.

[3] J. Dupeyroux, J. Diperi, M. Boyron, S. Viollet, and J. Serres, "A novel insect-inspired optical compass sensor for a hexapod walking robot," in 2017 IEEE/RSJ International Conference on Intelligent Robots and Systems (IROS). IEEE, 2017, pp. 3439-3445.

[4] J. Dupeyroux, S. Viollet, and J. R. Serres, "Polarized skylight-based heading measurements: a bio-inspired approach," Journal of the Royal Society Interface, vol. 16, no. 150, p. 20180878, 2019.

[5] -, "An ant-inspired celestial compass applied to autonomous outdoor robot navigation," Robotics and Autonomous Systems, vol. 117 , pp. 40 - 56, 2019.

[6] S. Mafrica, S. Godiot, M. Menouni, M. Boyron, F. Expert, R. Juston, N. Marchand, F. Ruffier, and S. Viollet, "A bio-inspired analog silicon retina with michaelis-menten auto-adaptive pixels sensitive to small and large changes in light," Optics express, vol. 23, no. 5, pp. 56145635, 2015.

[7] E. Vanhoutte, S. Mafrica, F. Ruffier, R. Bootsma, and J. Serres, "Timeof-travel methods for measuring optical flow on board a micro flying robot," Sensors, vol. 17, no. 3, p. 571, 2017.

[8] R. Wehner, "The desert ant's navigational toolkit: procedural rather than positional knowledge," Navigation, vol. 55, no. 2, pp. 101-114, 2008.

[9] R. Wehner, T. Hoinville, H. Cruse, and K. Cheng, "Steering intermediate courses: desert ants combine information from various navigational routines," Journal of Comparative Physiology A, vol. 202, no. 7, pp. $459-472,2016$.

[10] S. Sommer, C. von Beeren, and R. Wehner, "Multiroute memories in desert ants," Proceedings of the National Academy of Sciences, vol. 105, no. 1, pp. 317-322, 2008.

[11] A. Wystrach, S. Schwarz, P. Graham, and K. Cheng, "Running paths to nowhere: repetition of routes shows how navigating ants modulate online the weights accorded to cues," Animal cognition, vol. 22, no. 2 , pp. 213-222, 2019.

[12] A. Narendra, "Homing strategies of the australian desert ant melophorus bagoti ii. interaction of the path integrator with visual cue information," Journal of Experimental Biology, vol. 210, no. 10, pp. 1804-1812, 2007.

[13] S. Heinze, A. Narendra, and A. Cheung, "Principles of insect path integration," Current Biology, vol. 28, no. 17, pp. R1043-R1058, 2018.

[14] B. Cartwright and T. S. Collett, "Landmark learning in bees," Journal of comparative physiology, vol. 151 , no. 4, pp. 521-543, 1983.

[15] T. S. Collett and J. Zeil, Places and landmarks: An arthropod perspective. Oxford University Press, 1998.

[16] J. Zeil, M. I. Hofmann, and J. S. Chahl, "Catchment areas of panoramic snapshots in outdoor scenes," JOSA A, vol. 20, no. 3, pp. 450-469, 2003.

[17] R. Benosman, S. Kang, and O. Faugeras, Panoramic vision. SpringerVerlag New York, Berlin, Heidelberg, 2000.
[18] D. Lambrinos, R. Möller, T. Labhart, R. Pfeifer, and R. Wehner, "A mobile robot employing insect strategies for navigation," Robotics and Autonomous systems, vol. 30, no. 1-2, pp. 39-64, 2000.

[19] W. Stürzl and H. A. Mallot, "Efficient visual homing based on fourier transformed panoramic images," Robotics and Autonomous Systems, vol. 54, no. 4, pp. 300-313, 2006.

[20] H. G. Meyer, D. Klimeck, J. Paskarbeit, U. Rückert, M. Egelhaaf, M. Porrmann, and A. Schneider, "Resource-efficient bio-inspired visual processing on the hexapod walking robot hector," Plos one, vol. 15 , no. 4, p. e0230620, 2020.

[21] T. Goedemé, M. Nuttin, T. Tuytelaars, and L. Van Gool, "Omnidirectional vision based topological navigation," International Journal of Computer Vision, vol. 74, no. 3, pp. 219-236, 2007.

[22] M. Liu, D. Scaramuzza, C. Pradalier, R. Siegwart, and Q. Chen, "Scene recognition with omnidirectional vision for topological map using lightweight adaptive descriptors," in 2009 IEEE/RSJ International Conference on Intelligent Robots and Systems. IEEE, 2009, pp. 116-121.

[23] T. Lemaire and S. Lacroix, "Slam with panoramic vision," Journal of Field Robotics, vol. 24, no. 1-2, pp. 91-111, 2007.

[24] B. Metka, M. Franzius, and U. Bauer-Wersing, "Bio-inspired visual self-localization in real world scenarios using slow feature analysis," PloS one, vol. 13, no. 9, p. e0203994, 2018.

[25] L. Matuszyk, A. Zelinsky, L. Nilsson, and M. Rilbe, "Stereo panoramic vision for monitoring vehicle blind-spots," in IEEE Intelligent Vehicles Symposium, 2004. IEEE, 2004, pp. 31-36.

[26] P. Gaussier, C. Joulain, J.-P. Banquet, S. Leprêtre, and A. Revel, "The visual homing problem: An example of robotics/biology cross fertilization," Robotics and autonomous systems, vol. 30, no. 1-2, pp. 155-180, 2000.

[27] L. Ran, Y. Zhang, Q. Zhang, and T. Yang, "Convolutional neural network-based robot navigation using uncalibrated spherical images," Sensors, vol. 17, no. 6, p. 1341, 2017.

[28] E. Rigosi, S. D. Wiederman, and D. C. O'Carroll, "Visual acuity of the honey bee retina and the limits for feature detection," Scientific reports, vol. 7, p. 45972, 2017.

[29] M. Juusola and A. S. French, "Visual acuity for moving objects in first-and second-order neurons of the fly compound eye," Journal of neurophysiology, vol. 77, no. 3, pp. 1487-1495, 1997.

[30] C. Zollikofer, R. Wehner, and T. Fukushi, "Optical scaling in conspecific cataglyphis ants," Journal of Experimental Biology, vol. 198, no. 8, pp. $1637-1646,1995$.

[31] M. F. Land, "Visual acuity in insects," Annual review of entomology, vol. 42, no. 1 , pp. 147-177, 1997.

[32] B. D. Lucas, T. Kanade, et al., An iterative image registration technique with an application to stereo vision. Vancouver, British Columbia, 1981.

[33] J.-Y. Bouguet et al., "Pyramidal implementation of the affine lucas kanade feature tracker description of the algorithm," Intel Corporation, vol. 5, no. 1-10, p. 4, 2001. 